\title{
Role of the Tokamak ISTTOK on the EURATOM Fusion Programme
}

\author{
H. Fernandes, C.A.F. Varandas, and J.A.C. Cabral \\ Associação EURATOM/IST, Centro de Fusão Nuclear \\ Instituto Superior Técnico, 1049-001 Lisboa, Portugal
}

Received on 26 June, 2001

\begin{abstract}
This paper describes the role of the tokamak ISTTOK on the development of the portuguese fusion research team, in the frame of the EURATOM Fusion Programme. Main tasks on education and training, control and data acquisition, diagnostics and tokamak physics are summarized. Work carried out on ISTTOK in collaboration with foreign teams is also reported.
\end{abstract}

\section{Introduction}

ISTTOK is a small-size, large aspect ratio, limiter tokamak with an iron core transformer, in operation since 1993 at "Centro de Fusão Nuclear" (CFN), in the frame of the Fusion Programme of the European Atomic Energy Community (EURATOM). Its construction was started in mid-1990 reusing some components of the former TORTUR tokamak (support structure, vacuum vessel, copper shell, toroidal magnetic coils, transformer, capacitor banks, radiofrequency generator and discharge cleaning system). The other components of ISTTOK (vacuum and gas injection systems, poloidal windings and power supply for the toroidal B-field) as well as its diagnostics and control and data acquisition system were on-site designed and built [1].

ISTTOK ( $\left.R=46 \mathrm{~cm}, a=8.5 \mathrm{~cm}, B_{T}<3 \mathrm{~T}\right)$ has been very important for the creation and consolidation of the portuguese fusion research team by: (i) being an attraction pole for plasma physics students in an university environment; (ii) providing basic experimental formation in fusion- oriented plasma physics and technologies to young physicists and engineers; (iii) providing a test-bed for new diagnostic techniques as well as new VME digital instrumentation and systems; (iv) allowing to carry out a tokamak physics programme. More recently, the portuguese tokamak has been also used by foreign teams to perform particular experimental studies in collaboration with the ISTTOK team. The following sections of this paper contain short descriptions of the main ISTTOK components and activities, showing how this fusion experiment has contributed for the increase of the portuguese participation in the EU-
RATOM Fusion Programme.

\section{Education and training}

ISTTOK has been used to support the experimental part of post-graduation programmes on plasma physics and engineering. During the past eight years about twenty Master and two $\mathrm{PhD}$ thesis have been written based on work performed on this tokamak.

This programme of education and training has allowed CFN to create a research team with the adequate number of qualified professionals (about 20 in 1990 and 50 in 2001).

\section{Diagnostics}

ISTTOK is equipped with the following diagnostics: magnetic and electric probes, a microwave interferometer, a Thomson scattering system, a microwave reflectometer, a visible spectrometer, $\mathrm{X}$ - ray diagnostics, a laser-induced fluorescence system and a heavy ion beam diagnostic (Fig. 1) [1].

The work carried out on the development of these diagnostics has permitted the CFN staff to acquire internationally recognized expertise in several domains and to increase the number of requests for portuguese work in foreign projects. Presently CFN is responsible for the development of: (i) reflectometry systems for ASDEX Upgrade, TJ-II, MAST and ITER, based on ultra-fast broadband frequency sweeping, antenna located both in the lower and high magnetic field sides 
and new data evaluation techniques; (ii) multiple cell array detectors (MCAD) for the heavy ion beam diagnostic of the TJ-II stellarator, the first one using simultaneously two detection techniques for the secondary ions: electrostatic energy analyser and MCAD; and (iii) X-ray diagnostics for TCV. Portuguese engineers and physicists are still participating on the development of a laser induced fluorescence system for TJ-II, a Motional Stark Effect diagnostic for JET and Thomson scattering diagnostics for JET.

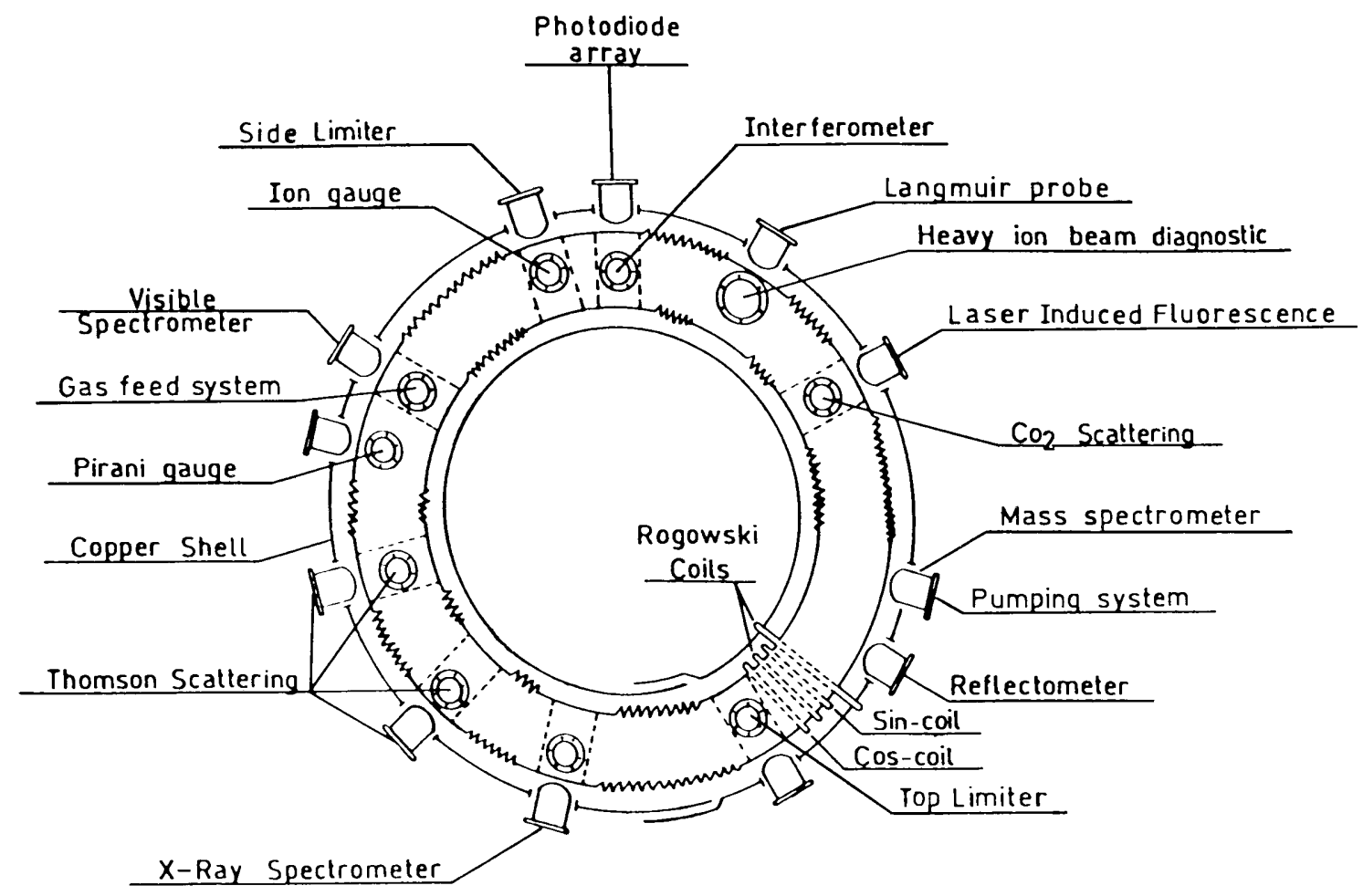

Figure 1. Main ISTTOK diagnostics.

\section{Control and data acquisition}

ISTTOK has a control and data acquisition system (SCAD) designed to comply with the requirements of the ISTTOK operation and research programme, in a distributed, modular, multivendor, integrated and transparent approach (Fig. 2) [2].

The computer system consists of several Pentium PCs, two CPU-30 (Motorola 68030) boards and one workstation, linked by an ethernet local area network, physically implemented by thin-wire cables and by a NOVELL server. The multivendor character of the
SCAD hardware leads to the existence of the following operating systems: MS-DOS, WINDOWS-NT, OS9 and UNIX. Transparent communications are guaranteed by IPX/SPX, and TCP/IP protocols. At a higher level, the compatibility of all systems is obtained by common network file system services. The slow control system is based on a commercial vacuum controller unit and on on-site developed hardware and software. Fast control is guaranteed by a locally developed VME timing unit [3]. 


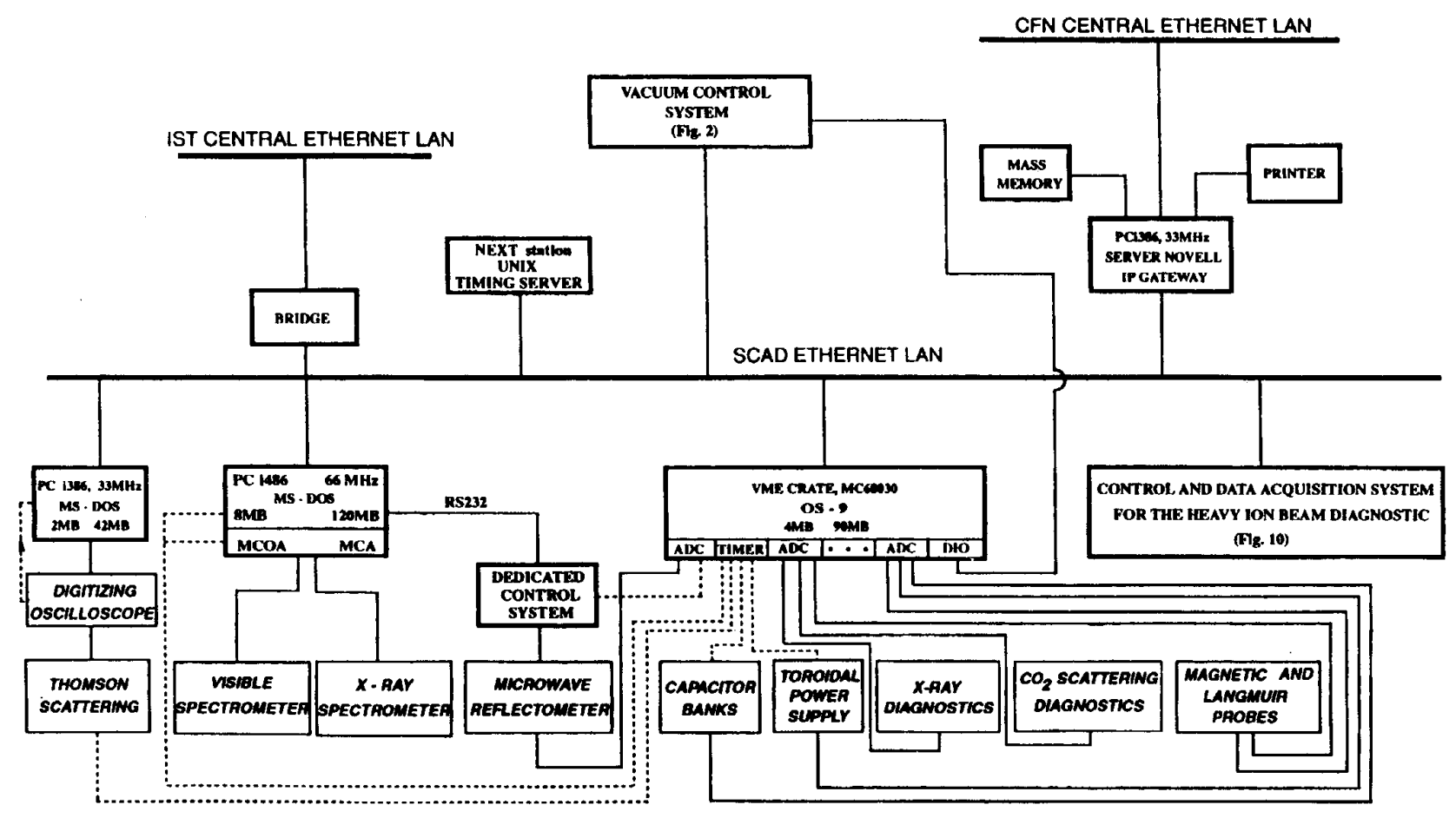

Figure 2. Block diagram of the ISTTOK control and data aquisition system.

The acquisition of the signals from the engineering and physics diagnostics is provided by on- site developed VME boards, commercial PC-based modules and a digitising oscilloscope. Table 1 presents the main characteristics of the CFN VME instrumentation [1]. The work carried out on ISTTOK has allowed creating expertise on the hardware for control and data acquisition. CFN is, or has been, responsible for the development of: (i) an interface amplifier and timing generator unit for the TCV X-ray pulse height analysis diagnostic [4]; (ii) the CAMAC and VME fast timing and event management systems for MAST [5]; (iii) the dedicated control and data acquisition system for the ASDEX Upgrade reflectometry diagnostic [6]; (iv) the dedicated control and data acquisition system for the JET correlation reflectometer [7]; (v) the monitoring system for the laser in-vessel viewing systems of JET and ITER-FEAT [8].

\section{Tokamak physics programme}

The ISTTOK plasma physics programme has been focussed in five main topics: (i) optimisation of the discharges; (ii) analysis of sawtooth discharges; (iii) study of the influence of limiter biasing on the plasma confinement and stability; (iv) studies on edge physics; (v) operation in alternating plasma current regime.
The scientific exploitation of this device has started by studying the influence of the external parameters on the tokamak discharges and testing the diagnostics efficiency. Changes in the primary voltage and transformer ratio have allowed attaining a wide range of plasma parameters. For high loop voltages discharges characterized by a plasma current of $11 \mathrm{kA}$ and duration of about $20 \mathrm{~ms}$, showing strong and rapid fluctuations, have been obtained. For intermediate loop voltages two types of very reproducible discharges have been attained: one, rather stable, with a current plateau up to $7 \mathrm{kA}$ lasting for about 40-60 ms (Fig. 3a) and another one showing sawtooth activity (Fig. 3b). For low loop voltages, very stable and long (> $60 \mathrm{~ms}$ ) discharges with a current plateau of about $3 \mathrm{kA}$ have been produced [9].

The study of sawtooth discharges has shown the importance of the new detection system proposed by the ISTTOK team for the heavy ion beam diagnostic, since it allows obtaining time- resolved plasma density profiles (Fig. 4) [9].

The influence of externally applied electric fields on the plasma confinement and stability has been studied by biasing the two diametrically opposed stainless steel limiters with DC [10] and $50 \mathrm{~Hz}$ voltages [11]. Experimental results have shown that positive (negative) bias has lead to a lower (higher) ratio of the plasma density 

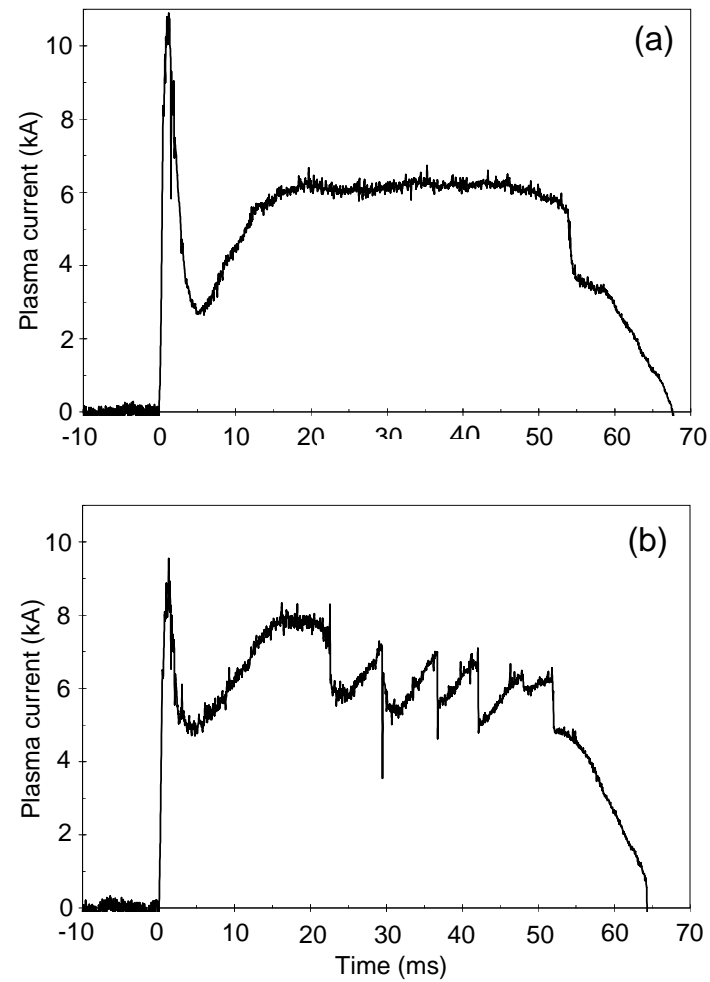

Figure 3. Evolution of the plasma current in two typical ISTTOK discharges obtained with medium loop voltage.
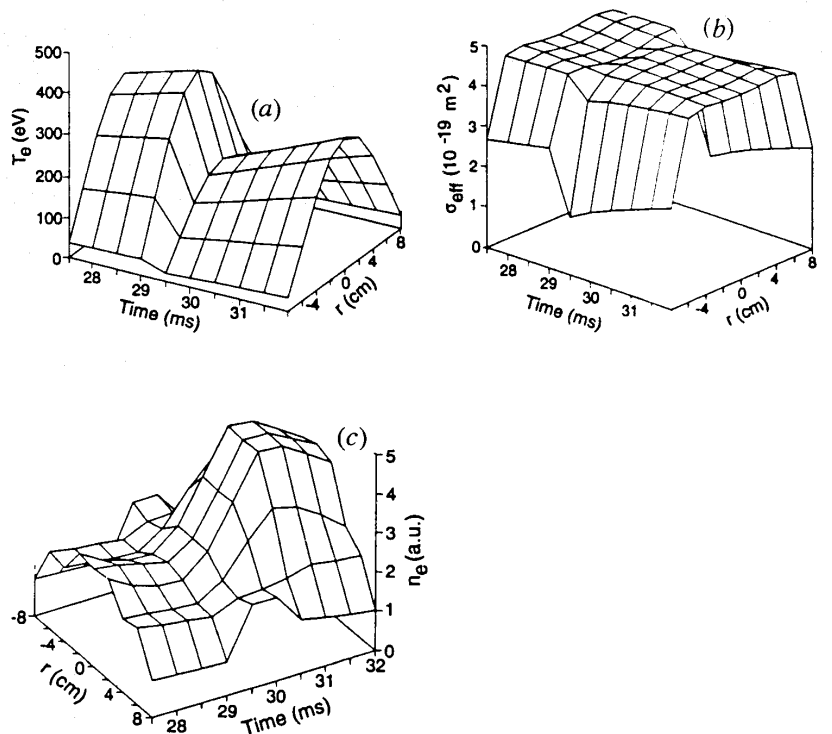

Figure 4. 3D representation of electron temperature (a) effective caesium second ionisation cross section (b) and plasma density (c) by the heavy ion beam diagnostic during a period of fast plasma current variation.

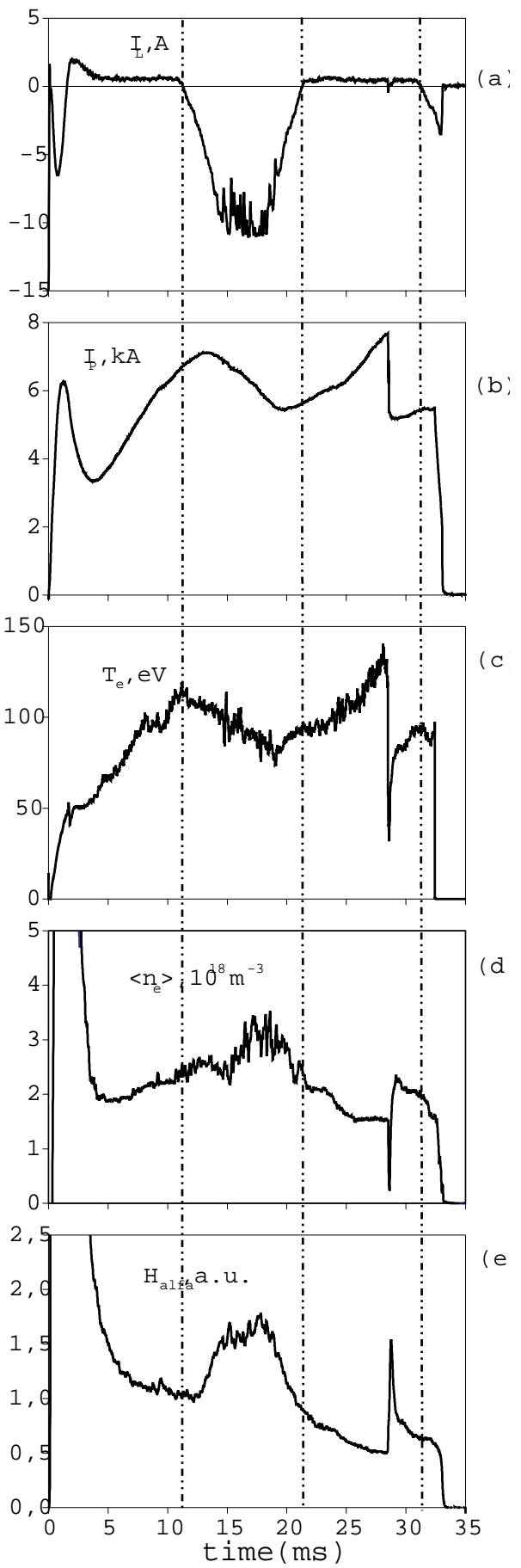

Figure 5. Temporal variation of the biasing current (a), Plasma current (b), electron temperature (c), average plasma density (d) and $H_{\alpha}$ radiation during a discharge with $50 \mathrm{~Hz}, \pm 115 V_{p p}$ limiter biasing. 


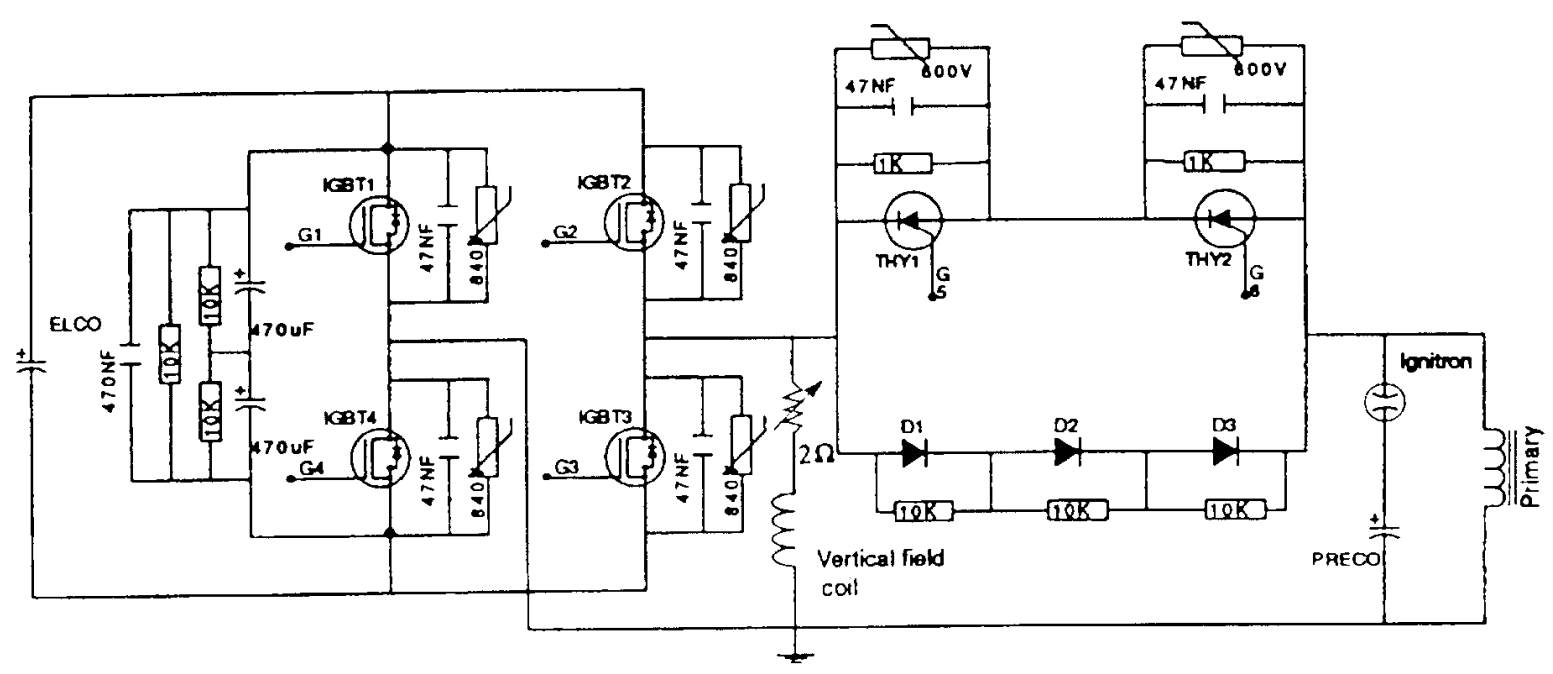

Figure 6. Scheme of the IGBT H-bridge power supply.

by the intensity of $H_{\alpha}$ radiation and to a degraded (improved) confinement (Fig. 5). Concerning the plasma stability positive (negative) bias has also lead to a poor (better) plasma column behaviour, revealed by an increase (decrease) of the magnetic as well as the electrostatic fluctuations, translated by an increase (decrease) of their spectral width and amplitude of the frequency of the dominant tearing modes. Further this work has shown that successful biasing experiments require voltages higher than 3-4 $\mathrm{kT}_{e} / \mathrm{e}$.

Alternating inductive plasma current operation has been performed using a specially designed power supply based on an H-bridge of four insulated gate bipolar transistors (IGBT) (Fig. 6) [12]. A maximum of seven flat tops half cycles, without dwell time was obtained, enlarging the duration of the discharges from 40 to 220 ms (Fig. 7). The plasma density has remained finite during current reversal [13].

\section{Collaboration with foreign Institutions}

Teams from CIEMAT (Spain) and the University of Innsbruck have carried out on ISTTOK respectively edge physics studies [14] and the measurement of the plasma potential by emissive Langmuir probes [15]. A new collaboration with Institute of Plasma Physics (Latvia), aiming at the implementation on the Portuguese tokamak of a liquid metal limiter, is now under discussion. Radial and poloidal electric field and plasma density fluctuations have been measured in the plasma boundary region using a multi-array of Langmuir probes in a triple configuration (Fig. 8). The radial profile of the Reynolds stress [16] shows a maximum gradient in the proximity of the location where the poloidal phase velocity of these fluctuations change sign (Fig. 9). The comparison of the magnitudes of flows induced by these fluctuation with those created by different mechanisms permits to conclude that Reynolds stress can drive significant poloidal flows in the plasma boundary region [16].

\section{Conclusions}

The role of small tokamaks in the Fusion Programme is recognized [17]. This paper shows how important the tokamak ISTTOK has been for the working programme of the Contract of Association between EURATOM and IST. Being the central core of the portuguese R\&D activities on Fusion, ISTTOK has given two important contributions for the EURATOM Fusion Programme: (i) Development of the R\&D activities on $\mathrm{AC}$ operation, heavy ion beam diagnostics and VME instrumentation for control and data acquisition, in which the ISTTOK team has a leading role; (ii) Education and training staff for the CFN team, allowing to increase the portuguese participation in foreign projects: ASDEXUpgrade, MAST, TCV, TJ-II, JET and ITER-FEAT. At a national level, ISTTOK has also been important for the increase of the acceptability of fusion as a new energy source for the next centuries. 

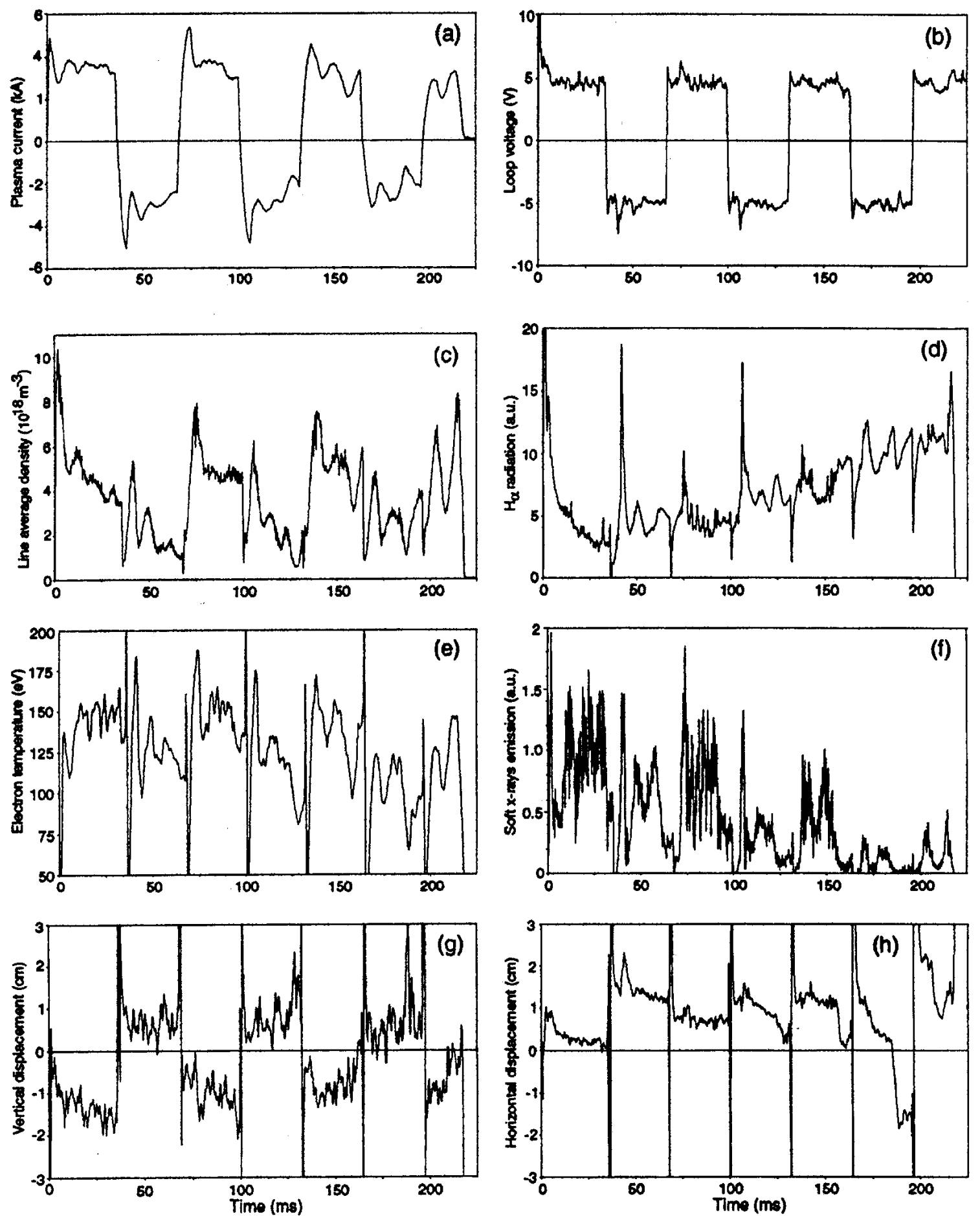

Figure 7. Evolution of the main discharge parameter in the AC shot $n^{0} 6043$ : (a) plasma current, (b) loop voltage, (c) line average electron density, (d) Ha radiation, (e) electron temperature, (f) soft X-ray emission, (g) vertical current axis displacement, $(\mathrm{h})$ horizontal current axis displacement. 


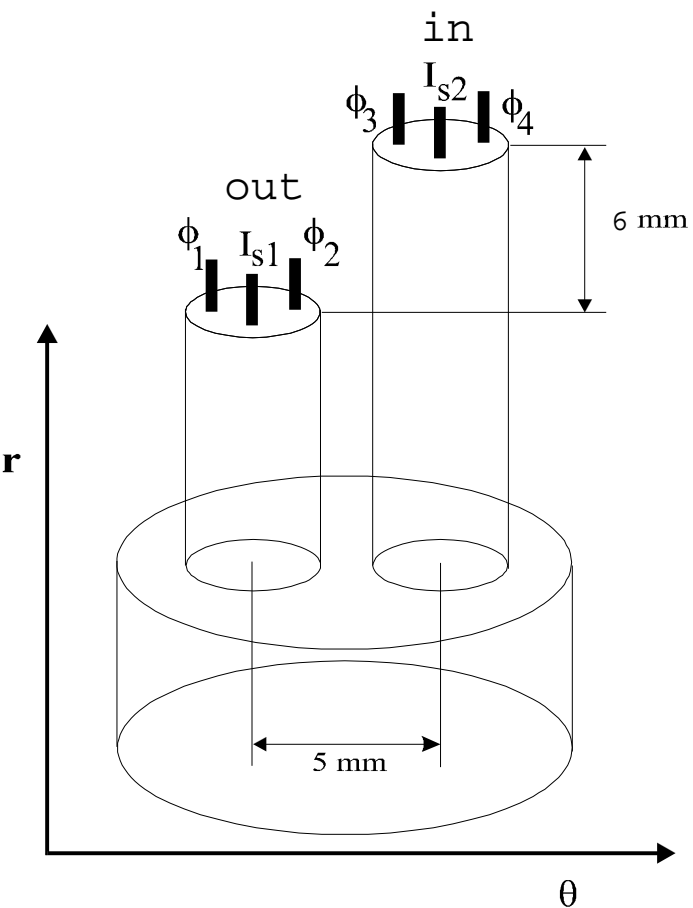

Figure 8. The triple Langmuir probe array.

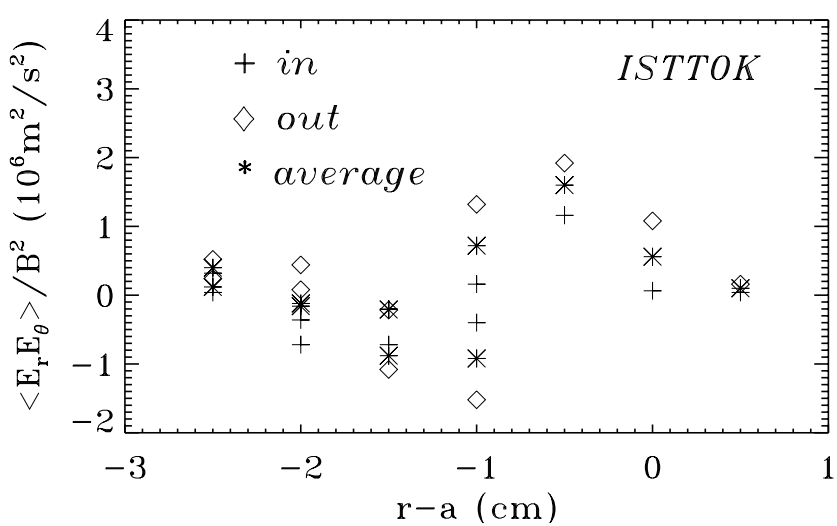

Figure 9. Radial profile of the electrostatic Reynolds stress in the plasma boundary of ISTTOK tokamak.

\section{Acknowledgments}

This work was carried out in the frame of the Contract of Association EURATOM/IST and has also received financial support from Fundaçãoo para a Ciência e a Tecnologia.

\section{References}

[1] C.A.F. Varandas, J.A.C. Cabral, J.T. Mendonça, M.P. Alonso, P. Amorim, B.B. Carvalho, C. Correia, L. Cupido, M.L. Carvalho, J.M. Dias, H. Fernandes, C.J. Freitas, S. Magalhães, A. Malaquias, M.E. Manso, A. Praxedes, J. Santana, F. Serra, A. Silva, A. Soares, J.
Sousa, W. van Toledo, P. Vaessen, P. Varela, S. Vergamota and Bart de Groot; Fusion Technology, 29, 105 (1996).

[2] C.A.F. Varandas, B.B. Carvalho, C. Correia, H. Fernandes, C. Freitas, J. Pires, J. Sousa and J.A.C. Cabral Nuclear Instruments and Methods in Physics Research A, 349, 547 (1994).

[3] C.A.F. Varandas, B. Carvalho, H. Fernandes, J. Sousa, C. Correia, M. Morgado, and J.A.C. Cabral. Review of Scientific Instruments, 66, 3383 (1995).

[4] J. Sousa, P. Amaro, P. Amorim, B. Duval and C.A.F. Varandas "19th Symposium on Fusion Technology", Lisboa, 16-20 Setembro 1996, 1, 881, Elsevier.

[5] J. Sousa, A. Batista, A. Combo, C. Correia, C.A.F. Varandas, D. Trotman and J. Waterhouse Fusion Engineering and Design, 43, 407 (1999).

[6] V. Grossman, J. Santos et al., Fusion Engineering and Design, 48, 25 (2000).

[7] N. Cruz, M. Correira, R. Pereira, L. Cupido, C. Correia, C.A.F. Varandas 3rd IAEA TCM on Control, Data Acquisition, and Remote Participation for Fusion Research, Padova 2001.

[8] R. Pereira, N. Cruz, C. Correia, C.A.F. Varandas 3rd IAEA TCM on Control, Data Acquisition, and Remote Participation for Fusion Research, Padova 2001.

[9] J.A.C. Cabral, C.A.F. Varandas, A. Malaquias, A. Praxedes, M.P. Alonso, P. Belo, R. Canrio, H. Fernandes, J. Ferreira, C.J. Freitas, R. Gomes, J. Pires, C. Silva, A. Soares, J. Sousa and P.H.M. Vaessen. Plasma Physics and Controlled Fusion, 38, 51 (1996).

[10] J.A.C. Cabral, C.A.F. Varandas, M.P. Alonso, P. Belo, R. Canrio, H. Fernandes, R. Gomes, A. Malaquias, P. Malinov, F. Serra, F. Silva and A. Soares. Plasma Physics and Controlled Fusion, 40, 1 (1998).

[11] J.A.C. Cabral, H. Figueiredo, I. Nedzelsky, C. Silva and C.A.F. Varandas "28th EPS Conference on Controlled Fusion and Plasma Physics", Funchal, 18-22 June, 2001.

[12] H. Fernandes, C.A.F. Varandas, J.A.C. Cabral, H. Figueiredo e R. Galvão Fusion Engineering and Design, 43, 101 (1998).

[13] H. Fernandes, H. Figueiredo, A. Soares, C.A.F. Varandas, J.A.C. Cabral, e a Equipa ISTTOK "24th European Physical Society Conference on Controlled Fusion and Plasma Physics", Berchtesgaden, 9-13 Junho 1997.

[14] C. Silva, H. Fernandes, H. Figueiredo, C.A.F. Varandas, J.A.C. Cabral, M.A. Pedrosa, C. Hidalgo e E. Snchez. "EU-UF Transport Task Force Workshop", Gothenburg, Suécia, 7 a 10 de Setembro de 1998.

[15] R. Schrittwieser, C. Avram, P.C. Balan, J.A.C. Cabral, F.H. Figueiredo, V. Pohoata and C.A.F. Varandas "4th International Workshop on Electrical Probes in Magnetised Plasmas", Berlin, Germany, 2000.

[16] C. Hidalgo, C. Silva, M Pedrosa, E. Sanchez, H. Fernandes, e C.A.F. Varandas Physical Review Letters.

[17] D.C. Robinson, Plasma Phys. Control. Fusion 35, B91. 